\title{
Téoros
}

Revue de recherche en tourisme

\section{Attractivité touristique et « sens " géo-anthropologique des territoires}

\section{Serge Gagnon}

Volume 26, numéro 2, été 2007

L’attractivité touristique des territoires

URI : https://id.erudit.org/iderudit/1070940ar

DOI : https://doi.org/10.7202/1070940ar

Aller au sommaire du numéro

Éditeur(s)

Université du Québec à Montréal

ISSN

0712-8657 (imprimé)

1923-2705 (numérique)

Découvrir la revue

Citer cet article

Gagnon, S. (2007). Attractivité touristique et « sens " géo-anthropologique des territoires. Téoros, 26(2), 5-11. https://doi.org/10.7202/1070940ar d'utilisation que vous pouvez consulter en ligne.

https://apropos.erudit.org/fr/usagers/politique-dutilisation/ 


\section{Attractivité touristique et "sens " géo-anthropologique des territoires}

\section{Serge Gagnon}

En tourisme, le premier problème concerne l'attractivité des territoires. L'attractivité d'un lieu ne serait pas donnée par la nature ni par la culture. Elle serait engendrée. Elle procéderait d'une émergence corrélée à un investissement de valeur de nature identitaire. Si l'on est attiré par un panorama, un paysage, il y a lieu de penser que le spectacle qui attire a été auparavant engendré par autre chose que les données naturelles et culturelles qu'il contient. Un paysage attire, par exemple, dans la mesure où quelque chose de plus, par rapport à ses contenus tangibles, a été investi. Ce quelque chose de plus, c'est une représentation symbolique, la plus généralement offerte par un ou des artistes.

Un nouveau rapport à l'espace est en voie d'émergence. Ce rapport se fonderait sur la saisie esthétique de certains lieux choisis, d'abord représentés en "paysages " par les artistes, puis transformés afin de ressembler justement à ces "paysages ", finalement destinés à des occupations de tourisme. Un lieu touristique serait donc attractif dans la mesure où un peintre, un écrivain, un poète, un orateur, un musicien, un photographe, l'aurait célébré au préalable. C'est ce qu'Alain Roger nomme «l'artialisation ». Façonnés par des regards multiples, les paysages aujourd'hui touristiques ont frappé l'imaginaire des premiers explorateurs, ils ont constitué des repères territoriaux, ont inspiré les artistes et ont été le théâtre d'aménagements prestigieux (château Frontenac à Québec).

Cet article aborde la problématique de l'attractivité ou ce qui fait qu'une position va attirer ou repousser des touristes. Ce qui est présenté constitue une nouvelle objec-

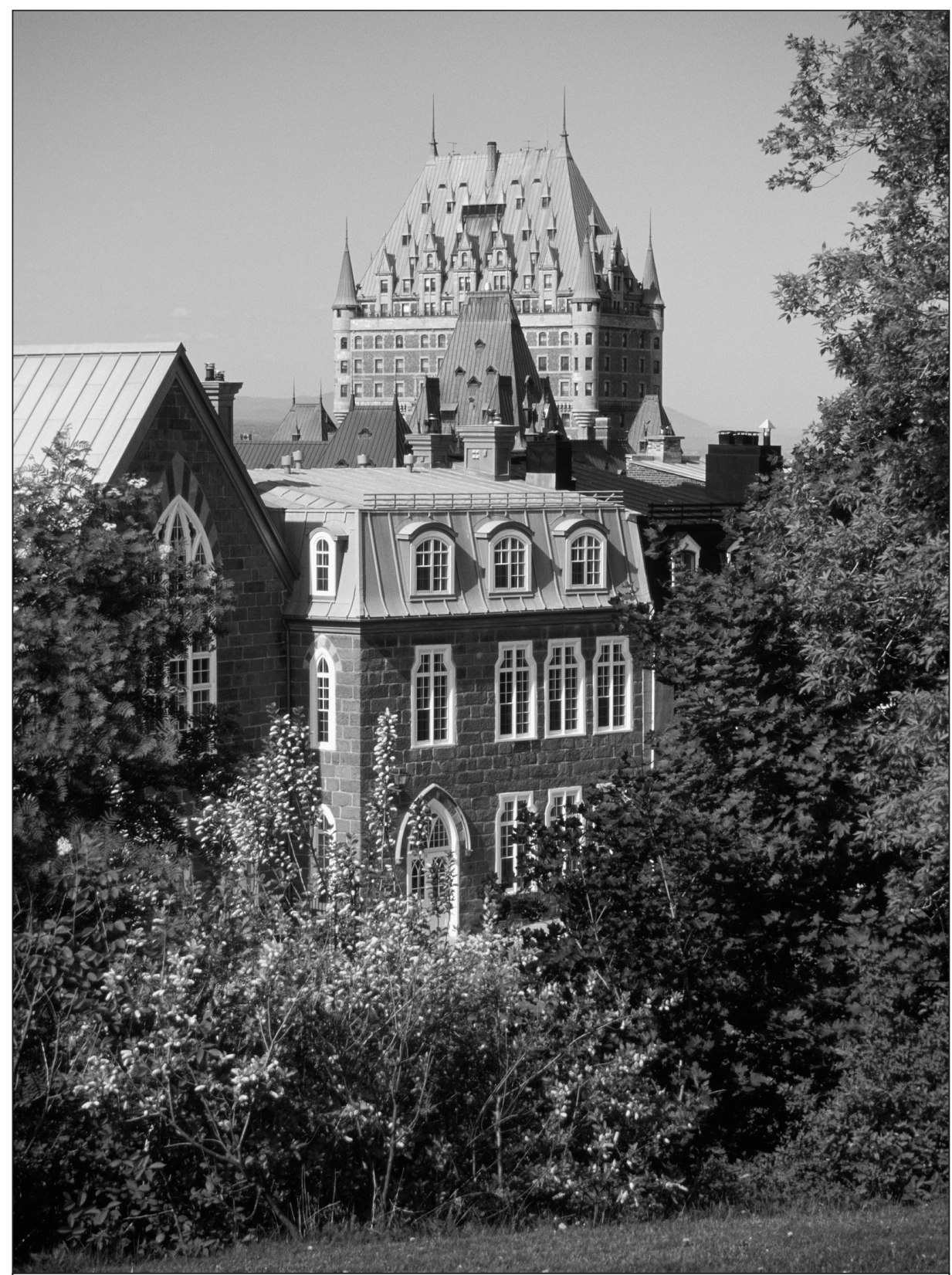

Château Frontenac, Québec.

Photo : Louise Mondoux, Tourisme Québec. 
tivité géographique concernant la manifestation sensible du tourisme (Gagnon, 2003). L'approche préconisée établit une distance par rapport aux interprétations usuelles qui réduisent explicitement ou implicitement l'attractivité des lieux à une spatialisation de contenus économiques contraints par les conditions naturelles ou anthropiques. L'objectif visé par ce texte consiste à ne pas fragmenter le regard porté sur le l'attractivité des positions et ainsi à transcender les descriptions et les explications sectorielles qui se sont multipliées ces dernières années, sans pour autant parvenir à constituer l'assise d'une véritable interprétation des processus qui sont à l'origine de l'attrait d'un lieu. L'article s'organise en trois parties: trois démarches typologiques utilisées pour distinguer l'attractivité touristique d'un territoire sont d'abord présentées ; vient ensuite l'énoncé d'une hypothèse géographique structurale (Desmarais, 2001; Desmarais et Ritchot, 2000) ; puis les rappels théoriques conduiront à exposer des exemples empiriques qui explicitent la pertinence du problème de l'attractivité dans l'organisation de certaines aires touristiques québécoises.

\section{Les regards classiques sur l'attractivité}

L'attractivité de certains lieux est généralement interprétée comme étant "the basic element on which tourism is developed" (Lew, 1987 : 554). Selon un spécialiste américain en la matière, Clare A. Gunn (1988: 37), "Because of the pull they exert on the traveller, all can be classified by the generic term attractions. [...] Without developed attractions tourism as we now know it could not exist. " La problématique des attractions touristiques est connue au Québec, surtout depuis les années 1970. Jean Stafford et Marcel Samson en évaluent le bien-fondé (1989: 13). Un chercheur américain, Allan A. Lew (1987), établit un cadre de référence sur les «attraits » touristiques. Pour sa part, après avoir consulté plusieurs études sur le sujet, Lew propose une typologie en trois catégories pour circonscrire la valeur attractive des sites touristiques: idiographic perspective?approche géographique; organizational perspective?approche aménagiste ; et cognitive perspective?approche sociologique. Par ailleurs, au dire de Marc Laplante (1983 ns sont indispensables car, sans elles, il est difficile de percevoir les lacunes, les trous, les éléments sous-développés de l'ensemble". II est donc pertinent de comprendre la valeur attractive de certains lieux. Nous basant sur la catégorisation de Lew, nous allons recenser les interprétations classiques et voir comment les divers spécialistes du tourisme abordent la classification des attraits.

\section{L'approche géographique}

La géographie classique d'inspiration vidalienne a reconnu le rôle des attractions dans l'organisation des sites et des territoires touristiques. Selon Raoul Blanchard (1960) et Roger Brière (1961-1962), les positions attractives jouent un rôle de premier plan dans l'organisation spatiale des territoires. Ceux-ci s'organiseraient à partir de pôles attractifs sur lesquels vient se brancher l'activité économique qui rentabilise les sites choisis. Chez Blanchard, les critères pour déterminer les localités touristiques passent d'abord par une identification des attractions naturelles et culturelles, pour ensuite prendre acte des infrastructures en place (1960 : 240).

Brière soutient que les déplacements " ne se font pas dans n'importe quelle direction ni vers n'importe quel site "; ils doivent être considérés "comme la réponse à une attraction ». Cet auteur établit d'ailleurs une distinction entre les sites qui attirent et ceux qui repoussent (1961-1962: 41). Brière précise en outre que les « attractions d'ordre physique" priment sur celles "d'ordre culturel ", ces dernières étant trop nombreuses pour être classées. L'approche empiriste d'un site attractif fait donc référence à ses qualités naturelles (panorama, faune, flore, climat, etc.) et culturelles (histoire, population, monument, institution, etc.).

Le géographe Jean-Pierre Lozato-Giotart nomme «sitologie touristique » l'opération qui consiste à détecter les attraits. II pose comme étant crucial le rôle des "faits naturels, [des] faits de civilisations et économiques [qui seraient] à l'origine de la motivation touristique tandis que les facteurs techniques [auraient] contribué à l'élargissement social et spatial du tourisme " (1993 : 40-72). Lozato-Giotart s'appuie sur des propositions élaborées en 1972 par Pierre Defert (1972: 3-13) : "Nous entendons par ressources tout élément naturel, toute activité humaine ou tout produit de l'activité humaine qui [peut] motiver un déplacement désintéressé. " À partir de cette prémisse, Defert classifie les attractions, qu'il nomme ressources et activités touristiques, selon quatre thèmes (hydrôme, phythôme, lithôme et anthropôme). Après avoir analysé les propositions de Defert, Marc Laplante (1983: 14) souligne que les efforts pour établir le "coefficient attractif d'une ressource " n'ont pas permis de définir l'attractivité des lieux.

\section{L'approche aménagiste}

L'approche aménagiste, qui remonte aux années 1970, concerne moins les caractères des sites que les attraits en soi. Le recours à l'échelle en constitue le fondement (Wall, 1996 : 242). En fonction des différentes échelles (locale, régionale, etc.), les types d'attraits touristiques sont rapportés à des conditions de possibilité, voire à des contextes: originalité, accessibilité, positionnement à l'égard de l'offre comparable, aménagement, planification, capacité de support du milieu, disponibilité en services, etc. Ce qui fonde l'attractivité du produit est alors lié à l'organisation de l'espace, à ce qui permet la mise en valeur d'un contenu préalablement évalué comme potentiel, quels que soient le lieu et les qualités naturelles ou culturelles de celui-ci.

Normand Cazelais (1999: 5-60) propose une lecture "volontaire ou aménagiste » de l'espace touristique québécois. Selon lui, les déplacements touristiques représentent un désir de se conjoindre avec un ailleurs attractif. Son argument se résume ainsi: tout peut être un attrait et provoquer des déplacements, par exemple la mer, une église, etc., cependant, ces phénomènes ont une "fonction première " qui n'est pas touristique. Le fait d'organiser un phénomène géographique peut lui permettre de devenir une attraction touristique, mais lui confère en l'occurrence une "fonction seconde» qui surdétermine la "fonction première ". Mais que signifient "attrait » et «attraction» touristiques? Selon Cazelais, l'attrait dépend de l'intention et de la portée données à un événement ou à un site, tandis que l'attraction réfère à un lieu aménagé et pensé dans le but déclaré d'attirer les gens. Tout peut être touristique, donc, mais, d'une part, le potentiel d'attractivité ne pourra se réaliser qu'en fonction de la distance des foyers émetteurs ou de l'accessibilité du site et, d'autre part, un signal, une marque humaine doit afficher l'intérêt. 
La distribution des usages, des infrastructures et des équipements répond du principe de rendement optimal. L'accent est mis sur les utilisations, les activités subordonnées et les aménagements qui les appuient. On réfère habituellement à la compatibilité des occupations, des usages et des activités strictement sous l'angle des nuisances. C'est le cas, entre autres, de la capacité de charge assujettie à des seuils destinés à préserver les équilibres écosystémiques (Thurot, 1980 ; Nadeau, 1999; Deprest, 1997). Un ensemble de normes conforte progressivement cette approche, de même que le savoir et les savoir-faire des aménagistes (Archambault, 1983 ; Gunn, 1988 ; 1997). On arrive même à donner le mode d'emploi pour créer une attraction (Gunn, 1997).

Du point de vue de la typologie aménagiste, la satisfaction des différents marchés, en particulier métropolitains, comme l'équité en matière de desserte et d'investissements, constituent des paramètres de base. Deux grandes catégories d'équipements touristiques sont envisagées: les uns sont tributaires de l'existence d'une ressource (centre de ski alpin, station balnéaire, lieu du patrimoine), tandis que les autres, qualifiés de footloose, sont liés à la proximité d'un marché (terrain de golf, parc thématique, casino). Les plans de développement des associations touristiques régionales témoignent de cette approche. L'analyse des encadrements administratifs et des potentiels conduit à un schéma directeur d'organisation territoriale qui répond à un certain nombre d'objectifs se rapportant aux particularités du milieu, de même qu'aux anticipations et aux attentes des acteurs. L'identification des aménagements et des équipements projetés ou souhaitables, l'élaboration d'un plan de commercialisation et l'esquisse de montages financiers complètent l'exercice de planification. Celui-ci donne lieu à d'importants travaux de cartographie des potentiels touristiques, comme à de nombreuses études sectorielles d'aménagement de stations et de régions touristiques, ainsi qu'à l'adoption, à la fin des années 1980, de plans de développement par chacune des associations touristiques régionales au Québec.

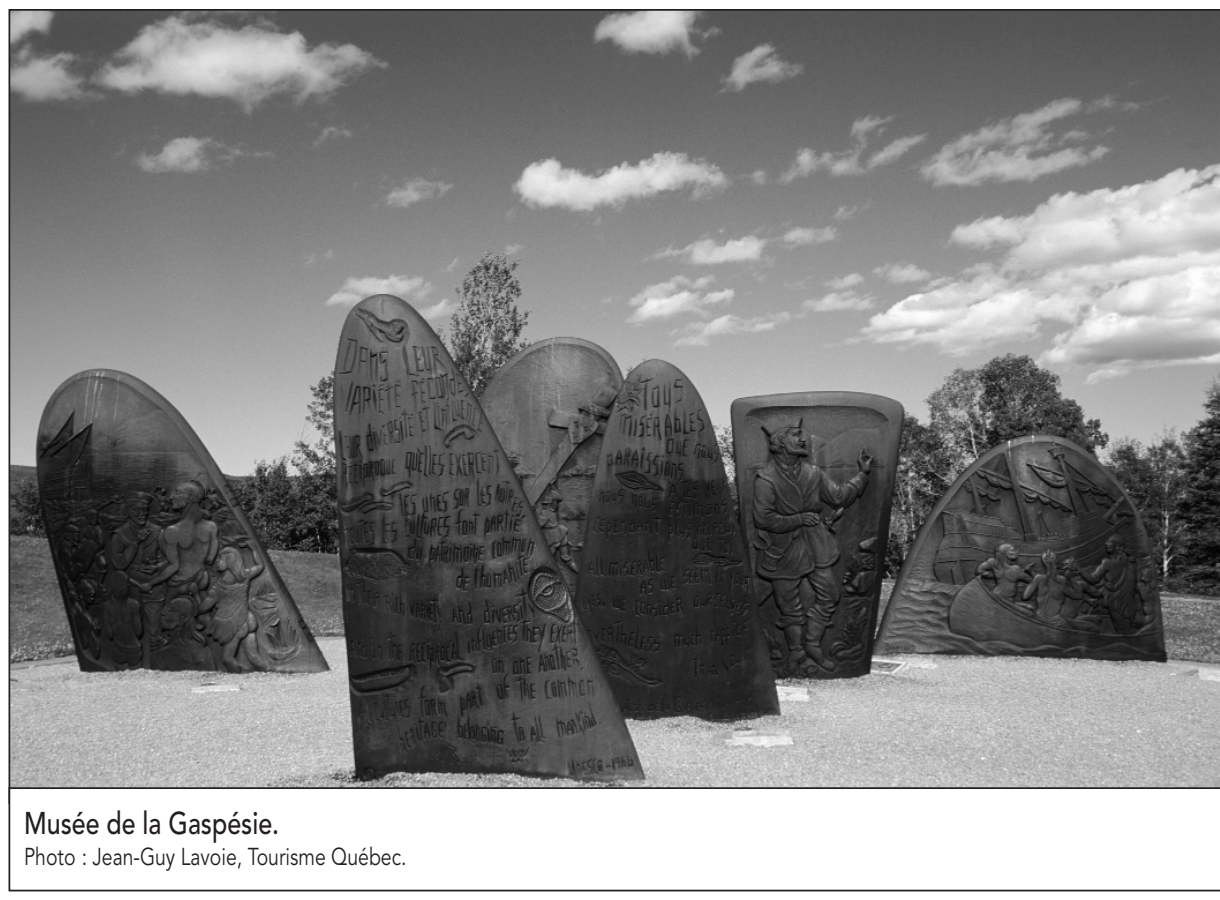

\section{L'approche sociologique}

Le troisième type d'approche en vue de discerner la valeur attractive des sites touristiques fait référence aux perceptions et aux expériences des touristes. L'attractivité dépend d'une quasi-connaissance de l'objet acquise par le touriste:

les inventeurs du tourisme moderne, dit MacCannell, ont emprunté aux religions l'essentiel de leurs technologies. Ils ont procédé, d'une part, à la sacralisation des sites et, d'autre part, ils ont mis au point des rituels d'approche [...] de façon telle que la progression vers l'attraction s'apparente à la montée vers un sommet, vers le temps fort recherché par le touriste: sa rencontre avec l'attraction (Laplante, 1983: 16).

L'approche perceptuelle est fondée en partie sur une étude de Dean MacCannell, qui définit une attraction touristique comme " an empirical relationship between a tourist, a sight, and a marker " (MacCannell, 1989 : 41). Marc Laplante et Louise Trottier (1985: 30-53; voir aussi Laplante, 1983 : 15-17) expérimentent cette méthodologie dans un ouvrage consacré aux attractions touristiques du Québec. Une attraction pourrait devenir effective à la condition qu'il y ait, d'une part, une "sacralisation" des lieux en cinq étapes - marquage, évaluation, enchâssement, reproduction mécanique et sociale - et, d'autre part, l'établis- sement d'un rituel d'approche de ces mêmes lieux. À la fin de leur analyse, les auteurs montrent que la "mécanique de base» du système de MacCannell est constituée par «l'interaction entre Marker, Sight et Tourist [...] le concept central de Sacralisation [étant] sous-jacent à cette interaction » (Laplante et Trottier : 53).

Neil Leiper (1990 : 370-382), de l'Université Massey en Nouvelle-Zélande, dresse un bilan des théories sur les attractions touristiques, allant jusqu'à proposer un modèle d'interprétation. Leiper adopte la définition de MacCannell. Une attraction touristique serait un système articulé à partir de trois éléments: "a person with touristic needs, a nucleus (any feature or characteristic of a place they might visit) and at least one marker (information about the nucleus) " (idem: 381). Pour Leiper, un touriste est un voyageur ou un visiteur qui cherche à vivre des expériences de loisir. Poussé par son propre désir de voyager, ce touriste est dirigé vers un "centre organisateur » ou nucleus par un réseau de marqueurs ou markers tout au long de son itinéraire. Ces marqueurs communiquent au touriste l'information sur le nucleus: avant son départ (generating marker), pendant son déplacement (transit marker) et finalement in situ (contiguous marker). Quant au nucleus, il représente " the central element in a tourist attraction system » (idem : 372). 
La méthode géostructurale se distingue des démarches typologiques que nous venons de résumer brièvement, bien que certaines propositions classiques soient porteuses d'intuitions convergentes, notamment à propos de la reconnaissance de l'attractivité d'un " centre organisateur ». La parenté entre la notion de «nucleus » et le concept géographique structural de «vacuum » est à cet égard remarquable (Ritchot, 1985, 1999; Desmarais 1992, 1995, 1998a, 1998b, 2001). Mis à part la méthode perceptuelle, les propositions classiques considèrent que l'attraction des lieux est fondée sur l'existence d'un certain «potentiel objectif» offert par les caractéristiques naturelles et culturelles d'un territoire. Par contre, Rémy Knafou et al. (1997 : 200-201) remettent en question la dimension empirique du recours à ces caractéristiques pour l'élaboration d'une typologie des attraits. Robert Soubrier (2000 : 244) souscrit à la même approche:

la difficulté majeure des concepteurs et chercheurs consiste à arriver à déterminer les éléments réellement attractifs, ceux qui constituent véritablement des sources de satisfaction. Jusqu'à présent, ces éléments ont été déterminés par la mesure du volume de participation ; un tel procédé ne nous semble cependant pas suffisant pour révéler et permettre de comprendre les raisons et les sources de satisfaction des utilisateurs.

Comment cerner une typologie des attraits et le rôle de ceux-ci dans la structuration d'un territoire? La typologie des attraits peut-elle se limiter à un inventaire des qualités physiques et culturelles? Les facilités d'accès et l'aménagement d'un site déterminent-ils sa typologie? L'attractivité d'un site touristique est-elle liée au seul marquage du territoire?

\section{Le sens géo-anthropologique d'une position touristique}

Dans la perspective de l'analyse structurale que nous préconisons, la définition géographique du tourisme passe effectivement par l'élaboration d'une typologie des attraits, dans la mesure cependant où les types de sites attractifs et leurs traits distinctifs suscitent certaines appropriations spatiales et les occupations conséquentes. Le problème de l'attractivité des lieux débouche ainsi sur une modélisation du phénomène en ce qu'il a de spatial. Tout espace touristique doit s'organiser autour de sites attractifs dont il s'agit de dégager les caractères et de comprendre la dynamique. Ces sites attractifs seraient stables et théoriquement connaissables de ce fait. Selon Jean-Michel Dewailly et Émile Flament (1993: 119),

L'observateur un peu attentif des répartitions spatiales et des rythmes touristiques ne peut manquer d'être frappé par l'existence d'un certain nombre de permanences. Elles s'expriment, notamment, par le prestige presque intact et l'attractivité conservée de certains lieux et stations fréquentés de très longue date [...] On pourrait presque parler de régions et de lieux centraux.

Dans cette optique de stabilité structurelle et de longue durée, le problème de l'attractivité des positions sélectionnées à des fins d'occupations touristiques peut faire appel à des investissements de valeurs identitaires et à leur communication par la gent artistique (écrivains, peintres, poètes, etc.). Le moment est venu de nous attarder à la réalité d'un investissement des valeurs identitaire dans l'espace, une saisie de nature géo-anthropologique. La formation de ces valeurs ne suffit pas à dynamiser un attrait (ou une répulsion). II faut en plus une actualisation spatiale, un investissement dans des positions qui, elles, sont attractives (ou répulsives). Les artistes auraient un rôle à tenir dans cette sémiogenèse (Desmarais, 1992).

\section{Les territoires du tourisme : des " objets de désir "}

Les territoires du tourisme constituent des objets de désir dont la valeur est investie et saisie affectivement. Marc Laplante, s'appuyant sur la théorie de Dean MacCannell, fait référence à l'affect, en spécifiant qu'une expérience touristique "doit atteindre certains points culminants de plaisir, d'émotions (peak experiments) " (dans Nadeau et Gilbert, 1988: 25). Pour expliciter la procédure d'investissement et de saisie des valeurs dans un lieu particulier, Gaëtan Desmarais se reporte à la sémiotique d'Algirdas Julien Greimas, conçue au cours des années 1970 et approfondie par Jean Petitot dans les années 1980 (Desmarais, 1998a; Petitot,
1988). Une sémiogenèse devancerait la spatialisation des "valeurs profondes" véhiculées par les sociétés à travers un processus génératif de signification.

Pour saisir la portée de la théorie sémiotique dans l'analyse géographique de l'attractivité, un retour à certaines remarques antérieures est bienvenu. Nous avons rapidement présenté le modèle de Neil Leiper concernant l'attractivité des lieux (supra). Celui-ci reconstitue un cheminement effectué par un tourist, qui est poussé par sa propre motivation, vers un centre organisateur ou nucleus. Des markers communiquent l'information sur le but à atteindre. Le touriste identifie ainsi l'attrait en se dirigeant vers le nucleus qui en est imprégné. Nous devons toutefois relativiser le volontarisme du tourist quant à son choix de destination. Le touriste est-il entièrement autonome dans ses décisions ? C'est à ce propos qu'entre en scène cette instance que Greimas appelle le «destinateur dit cosmologique». Le touriste prend l'initiative de se diriger de lui-même vers une position attractive, mais un "destinateur" le manipulerait dans cette voie. Selon nous, la procédure de «sacralisation » des lieux reconstituée par MacCannell pourrait renvoyer à cette sorte de manipulation. Quel est alors le rôle de l'agent destinateur dans le choix des lieux et, dans cette optique, comment l'investissement des valeurs et la saisie esthétique des positions touristiques procèdent-ils ?

Le destinateur cosmologique est l'agent responsable des valeurs, qui les investit dans des objets (territoire) et qui transmet au sujet (touriste) l'intentionnalité - le vouloir et le "devoir-faire »- de se conjoindre avec ces territoires (Desmarais, 1998a). Dans la situation qui nous intéresse et qui est dominée par la saisie esthétique ou affective des formes du monde sensible, le touriste va rejoindre les valeurs, non pas par le biais de la cognition, mais par celui de l'émotion. De là la référence à «l'affect».

Dans l'éclairage de ce cadre théorique, les positions du tourisme moderne sont des " objets de désir » en liaison avec une crise de la signification. Le « sujet esthétique est un sujet de désir dont les actions sont déterminées par [...] » les manifestations sensibles du monde naturel (montagne, mer, soleil, etc.) (Desmarais, 1998a: 91). 


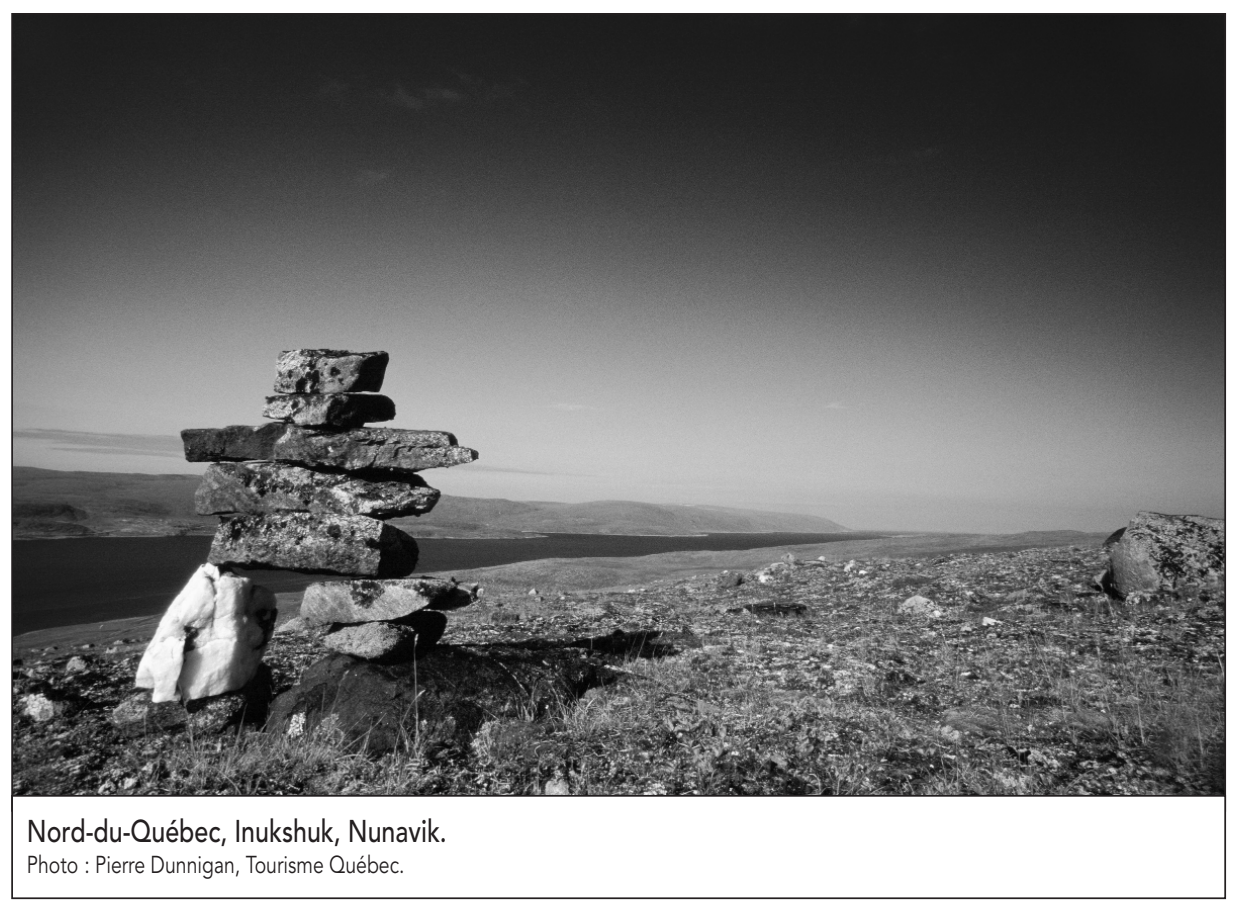

Qu'arrive-t-il avec le touriste? II part à la recherche des valeurs en contemplant les formes du monde sensible. Les valeurs virtuelles sont ainsi saisies esthétiquement, sur le mode de l'affect. "Le voyageur évolue de lieu en lieu, à la recherche de leur sens. Un sens que façonnèrent par tautologie, récits de voyage, œuvres poétiques et romanesques; un sens que les guides emprisonnèrent, figèrent, institutionnalisèrent en représentations stéréotypées, jusqu'à en faire des sites. " (Chadefaud, 1987 : 294)

C'est ainsi que, pour le touriste, la nature est porteuse de significations intrinsèques et son contact est recherché, non pas sur le mode cognitif, mais sur le mode affectif. À l'aide de ces mises au point, il est possible de reprendre le concept de "sacralisation » de MacCannell, ainsi que le modèle de Leiper, pour y intégrer l'instance "destinateur cosmologique». Le moment convient aussi pour introduire la question du romantisme. Le romantisme n'est pas seulement un courant artistique du XIX siècle. C'est aussi une réponse au problème de l'actualisation du "sens». Le romantisme est un mode particulier de saisie de la signification sans médiation transcendante. C'est la possibilité de saisir affectivement les valeurs profondes «à fleur de manifestation", pour reprendre une expression de Jean Petitot (1988). Qu'en est-il maintenant de la délégation, c'est-à- dire de la prise en charge, par un acteur concret, du rôle de destinateur cosmolomes géographiques affectivement saisies, le "destinateur délégué", du moins les premiers des destinateurs délégués, ce sont... les artistes. Dans le schéma de MacCannell, la "sacralisation » serait d'ailleurs assumée par ces artistes qui dès lors inspirent la production des markers.

À partir de ces éléments théoriques, il est maintenant possible de formuler une hypothèse concernant la définition sémiotique du tourisme. L'émergence du « destinateur cosmologique » aurait été constitutive de la signification profonde d'un tourisme "moderne» par définition, le "délégué» n'étant plus l'autorité qui persuade, mais le monde naturel qui fait désirer. La pratique touristique procède alors d'une saisie affective et esthétique de valeurs désormais captées à même les formes sensibles du monde environnant, les artistes jouant le rôle de transmission de ces valeurs, de délégués du destinateur cosmologique.

\section{Valeurs culturelles identitaires et modes d'investissement spatial}

Les valeurs culturelles, qui permettent aux touristes de s'identifier et qui sont «identitaires " de ce fait, ne sont pas produites par les destinateurs délégués, mais seulement communiquées. Les valeurs seraient gique? Si le celui-ci correspond aux for- senties, "vécues». Par ailleurs, cette communication ne repose pas sur un savoir imposé d'autorité, mais sur un affect. Les territoires touristiques seraient donc investis de valeurs culturelles prises en charge par les sociétés à des époques données, mais sans pour autant être entièrement décidées par ces sociétés. Les individus et les groupes sont dépositaires de valeurs culturelles qu'ils ne produisent pas pour autant dans leur totalité. Au surplus, ces valeurs constitutives d'identités individuelles et collectives sont communiquées par des positions géographiques; les formes du monde naturel (montagne, lac, vallée, etc.). Les touristes peuvent toujours investir des valeurs dans des positions, mais, d'une part, ces valeurs procèdent d'affects indécidables et, d'autre part, les positions actualisent les valeurs en question et dès lors «manipulent » les touristes.

D'un point de vue pratique, il convient de préciser le contenu de ces valeurs qui permettent de classifier les manifestations géographiques du tourisme. Au Québec, on a assisté depuis le $\mathrm{XIX}^{\mathrm{e}}$ siècle à une succession de phases lors desquelles furent communiquées des valeurs profondes. Ces grands systèmes de valeurs socioculturelles ont influencé l'aménagement d'équipements touristiques de surface (tableau 1).

II arrive, comme ce fut le cas au Québec et même en général, que les valeurs culturelles soient d'emblée véhiculées par les artistes avant d'être assumées par les touristes. Or, la prise sur la nature par l'artiste conduit à la production de "paysages" par le biais d'un processus d'" artialisation" (Roger, 1998; Beaudet, 1999). Comment procéderait "cette prise de possession de la nature par la culture» (Roger, $1998: 12$ ) ? Revenons à la saisie esthétique des valeurs socioculturelles réalisée par les touristes. Cette saisie procède des formes du monde sensible. Elle inspire l'adhésion à certaines valeurs, mais ce n'est pas dans la foulée d'une persuasion cognitive. La saisie esthétique, affective, fait plutôt appel aux expériences vécues. Elle se reconnaît, notoirement, dans les récits de voyages de JeanJacques Rousseau (Goulemot, 1986). Normand Cazelais (1999: 9) se livre à une interprétation similaire au sujet des autres corps artistiques: 
Tableau 1

Les grands systèmes de valeurs socioculturellesques au Québec

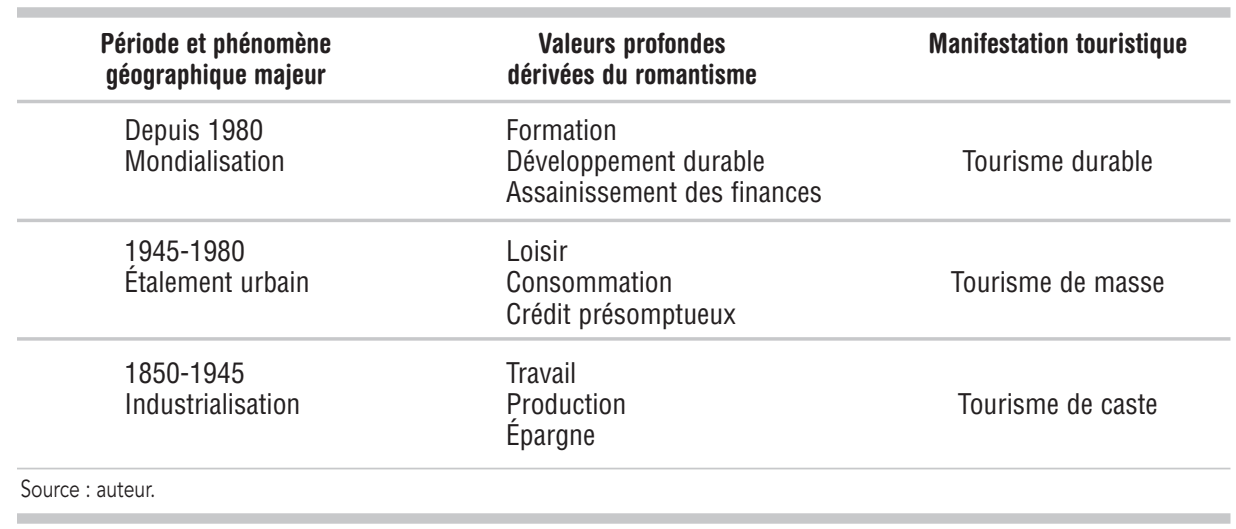

Et puis les tableaux du Tintoret et ses couchers de soleil sur Venise, de Cézanne qui reprenait, sans cesse épurées, les formes et la masse de la montagne SainteVictoire, de Gauguin qui, à grands aplats, recréait sur la toile les chaleurs exotiques de la Polynésie. Et même, les sons jungle de certaines pièces de Duke Ellington, les rythmes des chants et danses traditionnels de sa Hongrie natale que Liszt reprit dans plusieurs de ses œuvres. Écrits, visuels ou sonores, les paysages donnent le goût de partir, de voir - par soi-mêmeles vrais, sur place.

Si ces œuvres "donnent le goût de partir, de voir ", c'est bien que les œuvres artistiques sont des représentations de la conversion figurative et que les artistes sont des délégués du destinateur cosmologique; celui-ci correspond aux formes géographiques en elles-mêmes: les "beaux paysages"! La saisie des valeurs est bel et bien affective (émotionnelle) et non pas cognitive (intellectuelle). Ce qui revient à dire que le touriste se dirige vers le monde extérieur par l'émotion et non pas par le savoir. II composerait ainsi des impressions sur les formes qu'il perçoit. N'étant plus assuré de la vérité des valeurs (le savoir à ce stade est manquant), le touriste ferait son travail de réflexion à partir de la contemplation esthétique des choses. Roger Béteille (1988:35) montre comment les récits de voyages ou les œuvres littéraires, de la fin du XVIIIe siècle et du début XIX siècle, accordaient à la saisie affective la prépondérance sur la compréhension: «le paysage du tourisme est d'abord pré-senti dans l'imaginaire, puis senti [...] Le paysage du tourisme apparaît donc très largement comme un espace mythique ». Georges Cazes (1992: 77) renchérit: "L'observation des images prégnantes et dominantes dans une époque et une société données informe grandement sur les valeurs affectives et symboliques accordées à certains espaces, magnifiés et mythifiés ". II semble bien que ce furent des écrivains et des artistes qui, en contexte de romantisme révolutionnaire, assumèrent les premiers le rôle de transmettre le désir de se conjoindre aux valeurs.

\section{Tableau 2}

Les artistes et l'émergence paysagère de certaines aires touristiques au Québec

\begin{tabular}{lcccc} 
& $\begin{array}{c}\text { Émergence } \\
\text { paysagère }\end{array}$ & $\begin{array}{c}\text { Artiste-peintre } \\
\text { représentatif }\end{array}$ & $\begin{array}{c}\text { Première } \\
\text { appropriation }\end{array}$ & $\begin{array}{c}\text { Aire } \\
\text { touristique }\end{array}$ \\
\hline Outaouais & 1791 & Davies & 1800 & Ottawa-Hull \\
Charlevoix & 1850 & Bigby & 1854 & Pointe-au-Pic \\
Cantons de l'Est & 1840 & Bartlett & 1850 & Magog-Orford \\
Saguenay & 1860 & O'Brien & 1865 & Tadoussac \\
Laurentides & 1912 & Cullen & 1937 & Mont-Tremblant \\
Gaspésie & 1929 & Borduas & 1933 & Forillon-Percé \\
\hline
\end{tabular}

Source : auteur.

\section{Jalons d'artialisation au Québec et positions touristiques}

Nous retrouvons les thèses d'Alain Roger (1998: 17-18), relativement à la production des " paysages ". Le pays ne devient " paysage" que par un processus de double " artialisation " : in visu, c'est-à-dire dans et par le regard, et in situ, à savoir sur le terrain. En d'autres termes, la montagne, la mer et la campagne ne deviennent des paysages - des lieux privilégiés de tourisme - qu'en vertu d'un regard qui les produit littéralement, pour ne pas dire « littérairement". De ce point de vue, le "guide touristique est d'abord un véhicule artistique, un manuel d'artialisation" (idem: 119). L'investissement de valeur - une émergence paysagère - se traduit ultérieurement par des aménagements qui réalisent le paysage en refaçonnant le pays (Domon et al., 2000). Voici quelques jalons significatifs de cette "artialisation" en ce qu'elle a de particulier au Québec.

La production picturale aurait par la suite mobilisé les acteurs qui cherchaient à s'approprier les positions correspondant aux formes déjà imprégnées de valeurs. $\mathrm{Au}$ terme de cette appropriation, ces acteurs réalisaient la valorisation des sites en produisant le paysage conformément à sa représentation antérieure par l'art. Le paysage ne serait donc pas réductible au pays, à ses formes sensibles, à ses attributs de surface. Chaque paysage constituerait une saisie et un investissement de valeurs: une valorisation positionnelle. L'étude d'un territoire touristique et la détermination des types d'attraits qui y sont identifiés est alors inconcevable en l'absence d'une analyse des conditions de découverte et de valorisation "pionnière ". Les conditions sont posées au terme du processus d'investissement spatial que nous venons d'expliciter. Les valorisations d'un site touristique requièrent, par conséquent, au-delà du constat banal, une réflexion théoriquement responsable (Chadefaud, 1987 ; Debarbieux, 1992).

\section{Conclusion}

Le décryptage qualitatif de la valeur touristique des territoires montre que la lecture de ce phénomène socioculturel qu'est le tourisme est très complexe. L'interprétation des processus qui engendrent l'attractivité procède de sites investis 
euphoriquement et - fait intéressant célébrés par des artistes qui les ont fait désirer. Les territoires touristiques ne résultent donc pas de la projection de valeurs socioculturelles dans l'espace géographique, ni de l'insertion de celles-ci dans des structures socioéconomiques. Ces établissements procèdent plutôt de la saisie esthétique de prégnances affectives, investies dans des lieux privilégiés et qui agissent comme attracteurs. Ces attracteurs déterminent une dynamique d'appropriation qui confère un "sens " géoanthropologique à l'émergence des territoires touristiques. Lorsque les valeurs ont été investies dans telles et telles positions, celles-ci deviennent des lieux de convoitise pour les touristes et déclenchent ainsi la dynamique d'appropriation du potentiel d'attractivité. Cet éclairage neuf porté sur la question de l'attraction touristique des territoires pourrait servir la cause de l'organisation du développement régional des aires concernées, permettant ainsi de mieux cibler les interventions sur le terrain. Dans cette perspective, tout territoire n'est pas nécessairement touristique!

\section{Serge Gagnon est professeur au dépar-} tement de travail social et des sciences sociales de I'Université de Québec en

Outaonais.

\section{Bibliographie}

Archambault, Yves (1983), "Des outils et des intervenants ", Téoros, vol. 2, n 3, p. 2-5.

Beaudet, Gérard (1999), «Du jardin au paysage: le façonnement des lieux de villégiature et du tourisme au Québec », Téoros, vol. 18, n 1 , p. $14-25$.

Béteille, Roger (1988), "Le paysage, le mythe et le tourisme ", Acta Geographica, n 76, p. 35-41.

Blanchard, Raoul (1960), "Le tourisme», dans R. Blanchard (dir.), Le Canada français: Province de Québec, Étude géographique, Montréal, Fayard, p. 235-245.

Brière, Roger (1961-1962), "Les cadres d'une géographie touristique du Québec », Cahiers de Géographie de Québec, no 11, p. 39-64.

Cazelais, Normand (1999). "L'espace touristique québécois contemporain ", dans Normand Cazelais, Roger Nadeau et Gérard Beaudet (dir.), L'espace touristique, Québec, Les Presses de l'Université du Québec, p. 5-60.

Cazes, Georges (1992), Fondements pour une géographie du tourisme et des loisirs, Rosny, Bréal, collection « Amphi Géographie ».
Chadefaud, Michel (1987), Aux origines du tourisme dans les pays de l'Adour (Du mythe à l'espace: un essai de géographie historique), doctorat d'État, Département de géographie et d'aménagement de l'Université de Pau et des Pays de l'Adour.

Debarbieux, B. (1992), "La nature de 'l'explication géographique' dans l'analyse du tourisme ", dans Géographie économique et représentations, Paris, Anthropos/GIP RECLUS, p. 21-54.

Defert, Pierre (1972), Les ressources et les activités touristiques, Essai d'intégration, Les cahiers du CET (Centre d'études en tourisme) (série C : n 19), Université d'Aix-Marseille.

Deprest, Florence (1997), Enquête sur le tourisme de masse, l'écologie face au territoire, Paris, Mappemonde, Belin.

Desmarais, Gaëtan (1992), «Des prémisses de la théorie de la forme urbaine au parcours morphogénétique de l'établissement humain ", Cahiers de géographie du Québec, vol. 36, n 98, p. 251-273.

Desmarais, Gaëtan (1995), La morphogenèse de Paris, des origines à la révolution, Québec, L'Harmattan, Centre interuniversitaire d'études sur les lettres les arts et les traditions (CELAT).

Desmarais, Gaëtan (1998a), Dynamique du sens, Québec, Septentrion / CELAT.

Desmarais, Gaëtan (1998b), «Trois-conceptsclés pour les modèles morphodynamique de la ville ", Cahiers de géographie du Québec, vol. 42, n 117, p. 399-419.

Desmarais, Gaëtan (2001), « Pour une géographie humaine structurale ", Annales de Géographie, n०617, p. 3-21.

Desmarais, Gaëtan, et Gilles Ritchot (2000), La géographie structurale, Paris, L'Harmattan.

Dewailly, Jean-Michel, et Émile Flament (1993). Géographie du tourisme et des loisirs, collection «Dossier des images économiques du monde ", Paris, SEDES.

Domon, Gérald, Gérard Beaudet, et Martin Joly (2000), Évolution du territoire laurentidien : caractérisation et gestion des paysages, Université de Montréal, Chaire en paysage et environnement, Montréal, Isabelle Quentin Éditeur.

Gagnon, Serge (2003), L'Échiquier touristique québécois, Québec, Presses de l'Université du Québec, collection «Tourisme».

Goulemot, Jean-Marie (1986), « Le discours sur les voyages au XVIII siècle : remarques sur une émergence complexe ", Société, vol. 2, n 3 , p. 7-9.

Gunn, Clare A. (1988), Tourism Planning, Washington, Taylor \& Francis.

Gunn, Clare A. (1997) [3éd.], Vacationscape: Developing Tourist Areas, Washington, Taylor \& Francis.
Knafou, Rémy, Mireille Bruston, Florence Deprest, Philippe Duhamel, Jean-Christophe Gay, et Isabelle Sacareau (1997), "Une approche géographique du tourisme ", L'Espace géographique, nº 3, p. 193-204.

Laplante, Marc (1983), "Les attractions touristiques: un système à décoder ", Téoros, vol. 2, n², p. 14-22.

Laplante, Marc, et Louise Trottier (1985), L'évaluation des attractions touristiques au Québec : éléments de méthodologie, Université du Québec à Montréal, Centre de recherche en gestion et département d'études urbaines.

Leiper, Neil (1990), « Tourist Attraction Systems », Annals of Tourism Research, $n^{\circ} 17$, p. 367-384.

Lew, Allan A. (1987), "A Framework of Tourist Attraction Research ", Annals of Tourism Research, no 14, p. 553-575.

Lozato-Giotart, Jean-Pierre (1993) [4éd. ], Géographie du tourisme: De l'espace regardé à l'espace consommé, Paris, Masson, collection " Géographie ».

MacCannell, Dean (1989), «Introduction : Semiotic of Tourism ", Annals of Tourism Research, no 16, p. 1-6.

Nadeau, Roger (1999), «Tourisme et environnement ", dans Normand Cazelais, Roger Nadeau et Gérard Beaudet (dir.), L'espace touristique, Québec, Les Presses de l'Université du Québec, p. 61-131.

Nadeau, Roger, et Danielle Gilbert (1988), « Le tourisme de villégiature en Estrie » Téoros, vol. 7 , $\mathrm{n}^{\circ} 2$, p. 3-9.

Petitot, Jean (1988), « Un mémorialiste du visible, la quête du réel chez Proust », Protée, vol. 16, nos 1-2, p. 39-52.

Ritchot, Gilles (1999), Québec, forme d'établissement : étude de géographie régionale structurale, Paris, L'Harmattan.

Ritchot, Gilles, et C. Feltz, dir. (1985). Forme urbaine et pratique sociale, Louvain-laNeuve/Montréal, CIACO/Le Préambule.

Roger, Alain (1998), Court traité du paysage, Paris, Gallimard.

Soubrier, Robert (2000), Planification, aménagement et loisir, Québec, Presses de l'Université du Québec.

Stafford, Jean, et Marcel Samson (1989), «Évolution de l'industrie touristique et émergence de la recherche en tourisme au Québec ", La recherche en tourisme, Montréal, collection "Téoros: Les Fascicules », n 4, p. 10-15.

Thurot, J.M. (1980), Capacité de charge et production touristique, Aix-en-Provence, Centre des hautes études touristiques, Études et mémoires, $n^{\circ} 43$.

Wall, G. (1996). "Tourism Attractions: Points, Lines, and Areas", Annals of Tourism Research, vol. 23, p. 240-243. 